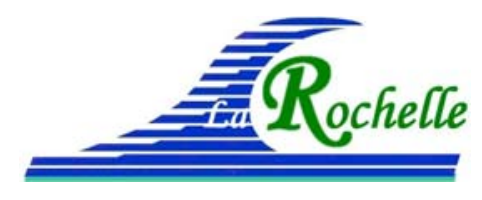

XVèmes Journées Nationales Génie Côtier - Génie Civil

La Rochelle, 29 au 31 mai 2018

DOI:10.5150/jngcgc.2018.048

(c) Editions Paralia CFL

disponible en ligne - http://www.paralia.fr - available online

\title{
Propagation de la houle à travers un milieu poreux : surface spécifique, disposition et paramètres de forme du poreux
}

\author{
Flora DESMET ${ }^{1,2}$, Christian RAFFOURT ${ }^{1}$, Antoine MANGIN ${ }^{1}$
}

1. ACRI-IN, 260 Route du Pin Montard, Sophia-Antipolis, 06410 Biot, France.

flora.desmet@gmail.com ; christian.raffourt@acri-in.fr ; antoine.mangin@acri.fr

2. INSA Lyon, Département de Génie Mécanique, 20 Avenue Albert Einstein, 69100

Villeurbanne, France.

\section{Résumé :}

Les structures protectrices dissipant la houle qui arrive sur la côte sont diverses et communément constituées de milieux poreux comme les digues en enrochements. Afin d'évaluer l'efficacité de telles structures, il est important de quantifier le taux de dissipation à l'aide des coefficients de transmission et de réflexion.

Dans ce projet, un canal à houle avec un milieu poreux est reproduit en utilisant le logiciel open source OpenFOAM. Les phénomènes de transfert entre énergie cinétique et énergie potentielle et de dissipation visqueuse sont modélisés par une méthode RANS (Reynolds-Averaged Navier-Stokes) en VOF (Volume of Fluid).

La confrontation de mesures physiques obtenues antérieurement aux résultats du modèle numérique permet de valider ce dernier sans recours à un calage spécifique.

Le modèle numérique complète les études expérimentales car il présente quelques avantages : temps de mise en œuvre et coût moins élevés, pas de problème d'échelle réduite pouvant induire des soucis délicats de similitude pour ces phénomènes d'ondes gravitaires avec dissipation visqueuse non négligeable, précision satisfaisante sans étalonnage préalable, pas de contrainte d'appareil de mesures. L'étude de l'importance de l'influence de la surface spécifique sur la dissipation est poursuivie grâce à ce moyen numérique. Comme pour l'étude précédente en canal physique, un milieu poreux composé d'un réseau de cylindres est disposé dans un canal "numérique". On présente les résultats de l'étude portant sur l'impact des paramètres de forme et d'arrangement, que l'on rapproche de l'influence constatée de la surface spécifique.

\section{Mots-clés :}

Houle, Milieu poreux, Dissipation, Réflexion, Surface Spécifique, OpenFOAM, RANS, VOF. 


\section{Thème 4 - Ouvrages portuaires, offshore et de plaisance}

\section{Introduction}

Le littoral est souvent fragilisé et abîmé par l'énergie des vagues venant se briser. Depuis des décennies, des ouvrages protecteurs ont été pensés afin de dissiper la houle arrivant sur la côte et de limiter les dommages. Ces ouvrages protecteurs sont souvent des milieux poreux à perméabilité élevée autorisant la dissipation visqueuse de l'énergie de l'onde gravitaire de houle par la génération d'écoulements turbulents au sein des pores. Ils ont été étudiés à de nombreuses reprises, notamment leurs coefficients de transmission et de réflexion. Le travail de recherche de CALHOUN (1971), portant sur le brise-lame du port de Monterey en Californie, peut être considéré comme l'approche scientifique pionnière dans ce domaine. Son travail a mis en lumière le rôle des ouvrages perméables dans la dynamique côtière des vagues, générant jusqu'à $40 \%$ de réflexion de la houle et environ 10 à $20 \%$ de transmission. Des interférences et des réflexions multiples se produisent à l'intérieur du milieu poreux menant à de la dissipation d'énergie. MADSEN (1983) a développé un des premiers modèles théoriques de la réflexion linéaire des vagues par un milieu poreux en eau peu profonde. Dans ce modèle, le coefficient de réflexion dépend des paramètres de la houle incidente et des propriétés de la paroi poreuse telles que sa largeur et sa porosité - rapport entre le volume des pores et le volume total du milieu poreux. ARNAUD (2016) a introduit la notion de surface spécifique - surface de contact fluide/solide par unité de volume pour étoffer la description d'un milieu poreux. Selon ses études expérimentales et analytiques, la surface spécifique semble jouer un rôle dans le processus de dissipation des milieux poreux. Cette influence est de nouveau étudiée dans le présent article. D'autres travaux ont été réalisés au sujet de la dissipation ayant lieu dans des réseaux de cylindres émergents. En effet, LIU et al. (2015) ont déterminé expérimentalement les coefficients de traînée d'un cylindre dans un tel réseau soumis à différentes houles. La plupart des modèles sont basés sur la théorie linéaire potentielle des ondes, et tiennent compte de la dissipation sous forme linéaire ou quadratique. Dans cet article, une approche non-linéaire est considérée, utilisant le modèle numérique RANS (Reynolds Averaged Navier-Stokes) pour étudier le comportement des écoulements fluides turbulents de la houle au sein du macroélément poreux. LAMBERT (2012) a étudié l'influence de la taille des mailles des éléments finis sur la propagation de la houle dans un canal numérique réalisé avec le logiciel OpenFOAM (OF). Il en ressort la nécessité d'avoir un maillage suffisamment fin pour ne pas dissiper la houle se propageant en condition laminaire dans le canal vide. Un équilibre entre précision et temps de calcul a été trouvé afin d'obtenir les résultats présentés dans cet article. Dans la première partie de cette communication, les réglages numériques permettant d'avoir un réseau de cylindres dans un canal numérique, ainsi que des conditions aux limites adéquates pour la génération et l'absorption de la houle, sont décrits. La deuxième partie présente les résultats numériques avec d'un côté, la reproduction des essais expérimentaux d'ARNAUD (2016) focalisée sur la dissipation, l'élévation de la surface libre et la 


\section{XVèmes Journées Nationales Génie Côtier - Génie Civil \\ La Rochelle, 29 au 31 mai 2018}

vitesse horizontale des particules d'eau et, de l'autre côté, l'influence de nouvelles dispositions et formes de cylindres sur la transformation de la houle.

\section{Mise en place du modèle numérique}

\subsection{Géométrie}

Les caractéristiques géométriques du modèle visent à reproduire le canal physique Sea Tech de Toulon, utilisé lors du travail expérimental d'ARNAUD (2016). Le canal numérique, à fond plat, a une profondeur de $0,3 \mathrm{~m}$ et une longueur de $13 \mathrm{~m}$ - au lieu de $10 \mathrm{~m}$ pour le canal physique - ceci afin d'éviter tout phénomène de réflexion en bout de canal qui pourrait venir perturber la mesure. Les effets de bord du milieu poreux sont négligés ainsi que l'interférence des sillages parallèles ce qui permet de modéliser seulement un couloir de $0,036 \mathrm{~m}$ de large par symétrie - bande jaune sur la figure 1-. Les milieux poreux utilisés pour la validation du modèle numérique sont conformes aux réseaux de cylindres utilisés par ARNAUD - cylindres de révolution disposés verticaux en quinconce. Comme la porosité est maintenue constante, le changement de diamètre des cylindres entraîne une modification du nombre de cylindres qui composent le milieu poreux de $1,2 \mathrm{~m}$ de long $(\mathrm{D}=0.020 \mathrm{~m}, \mathrm{~N}=300 ; \mathrm{D}=0.032 \mathrm{~m}, \mathrm{~N}=120 ; \mathrm{D}=0.050 \mathrm{~m}, \mathrm{~N}=50)$. De cette manière, la surface spécifique évolue, tout en gardant une porosité constante. La surface spécifique $s$ diminue lorsque le diamètre augmente et vaut respectivement $s=52.36,33.51$ et $21.82 \mathrm{~m}^{-1}$ pour les diamètres $\mathrm{D}=0.020,0.032$ et $0.050 \mathrm{~m}$. Dans la seconde partie de cette étude, la forme et la disposition des cylindres sont modifiées, tout en gardant la surface spécifique et la porosité, égales à celles du réseau initial de cylindres de diamètre $\mathrm{D}=0.032 \mathrm{~m}$. Plusieurs configurations sont étudiées : disposition en ligne dans la direction longitudinale puis transversale, cylindres en position horizontale, éléments de section carrée orientés de front puis de biais (figure 2).

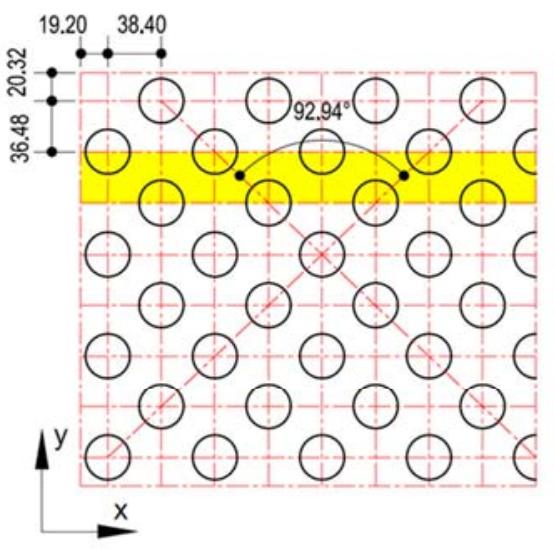

Figure 1. Paramètres géométriques du réseau de cylindres et trace en jaune $d u$ canal numérique

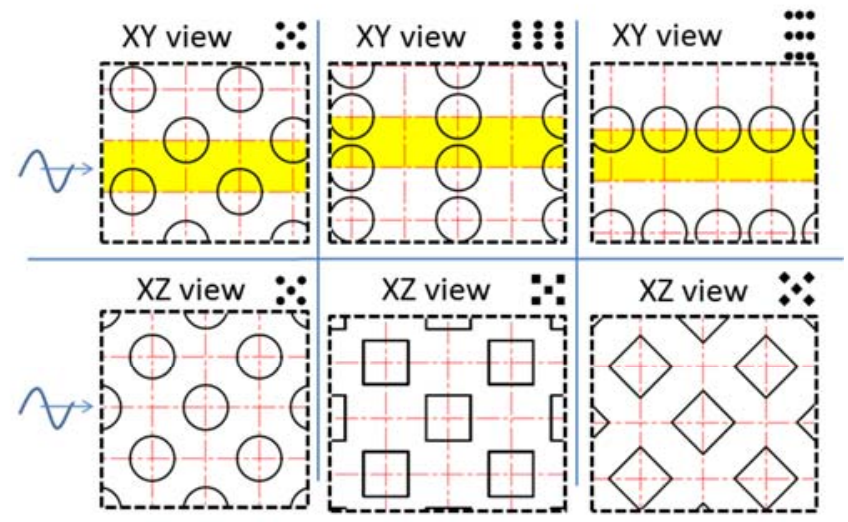

Figure 2. Forme de la section des cylindres, arrangement des motifs des réseaux (motifs horizontaux en haut avec emprise du canal numérique en jaune, motifs verticaux en bas) et symboles associés au-dessus des motifs 


\section{Thème 4 - Ouvrages portuaires, offshore et de plaisance}

\section{2 $\underline{\text { Simulation }}$}

Le maillage du canal numérique contenant le milieu poreux a été réalisé en utilisant deux types de mailleurs. Les maillages résultants sont comparés afin de sélectionner le plus efficace et vérifier que les résultats numériques ne sont pas induits par un artefact numérique dû au maillage. Pour la résolution, on exploite le solveur interFoam, destiné aux modèles comportant deux fluides incompressibles. La méthode VOF, basée sur une fonction discrète couplée à un schéma d'advection, permet de suivre le mouvement de l'interface entre les deux fluides, i.e. la surface libre. Le modèle de Stokes du deuxième ordre est implémenté pour produire la houle en entrée du canal (1). C'est un modèle approprié pour la génération de vagues sinusoïdales, que l'on rencontre généralement en eau profonde. $\mathrm{Du}$ fait que le batteur utilisé lors des expérimentations de référence génère de la houle sinusoïdale, pour les modélisations numériques le modèle de Stokes est utilisé bien que le canal reproduise une condition d'eau peu profonde. Que ce soit dans le canal physique ou bien numérique, les vagues se transforment naturellement en houle cnoïdale lors de leur propagation du fait de la faible profondeur.

$$
\eta=\frac{H}{2} \cos (k x-\sigma t)+\frac{H^{2} k}{16} \frac{\cosh (k h)}{\sinh ^{3}(k h)}(2+\cosh (2 k h)) \cos 2(k x-\sigma t)
$$

où : $\sigma$ est la fréquence de l'onde $\sigma=2 \pi / \mathrm{T}(\mathrm{rad} / \mathrm{s}), \mathrm{k}$ le nombre d'onde $\mathrm{k}=2 \pi / \lambda(\mathrm{rad} / \mathrm{m})$, $\mathrm{H}$ la hauteur de vague $(\mathrm{m})$, h la hauteur d'eau au repos $(\mathrm{m})$ et $\mathrm{x}$ la distance par rapport à l'entrée du canal.

A l'extrémité du canal, un modèle d'absorption en eau peu profonde est implémenté pour remplacer la plage dissipative fréquemment utilisée dans les bassins physiques. Cette plage a pour but de dissiper l'énergie des vagues en bout de canal et d'éviter ainsi leur réflexion. Un modèle de turbulence est appliqué, notamment sur le fond du canal ainsi que sur les parois des cylindres constituant le milieu poreux. La taille du maillage autour de ces surfaces est adaptée au modèle utilisé. Un modèle de faible nombre de Reynolds - k- $\omega$ SST - est implémenté. Cela nécessite d'avoir une distance adimensionnelle à la paroi $\mathrm{y}^{+}$comprise entre zéro et cinq, afin de contenir le centre de la maille la plus proche de la paroi dans la sous-couche laminaire. De cette manière, il est possible de représenter finement le comportement visqueux du fluide dans la couche limite turbulente, et ainsi de recueillir des valeurs de force de traînée et de perte de charge pertinentes. Trois houles de périodes différentes sont utilisées pour valider le modèle numérique sur trois réseaux poreux distincts, chacun composé d'un nombre de cylindres et d'un diamètre différents. La cambrure de la houle $(\varepsilon=0.01)$ ainsi que la profondeur d'eau au repos $(\mathrm{h}=0.23 \mathrm{~m})$ restent constantes, c'est pourquoi le changement de période induit une modification de la hauteur de vague. Le tableau 1 regroupe les données caractéristiques des trois houles. 


\section{XVèmes Journées Nationales Génie Côtier - Génie Civil \\ La Rochelle, 29 au 31 mai 2018}

Tableau 1. Caractéristiques des houles utilisées pour la validation du modèle numérique.

\begin{tabular}{ccc}
\hline Période & Longueur d'onde & Hauteur de vague \\
\hline$T=1.2 \mathrm{~s}$ & $\lambda=1.61 \mathrm{~m}$ & $H=0.017 \mathrm{~m}$ \\
$T=1.5 \mathrm{~s}$ & $\lambda=2.10 \mathrm{~m}$ & $H=0.021 \mathrm{~m}$ \\
$T=2 \mathrm{~s}$ & $\lambda=2.89 \mathrm{~m}$ & $H=0.029 \mathrm{~m}$ \\
\hline
\end{tabular}

\section{Résultats numériques}

\subsection{Validation}

Afin de valider le modèle numérique, dans toutes les configurations géométriques étudiées par ARNAUD (2016), plusieurs variables ont été étudiées, en particulier l'élévation de la surface libre, la vitesse horizontale des particules d'eau et les coefficients de transmission et de réflexion du milieu poreux. ARNAUD (2016) propose une normalisation de ses résultats. Ceux obtenus pour un réseau de cylindres de diamètre $\mathrm{D}=0.032 \mathrm{~m}$ soumis à la première houle du tableau 1 sont reportés en figure 3 et confrontés aux résultats du modèle OF.

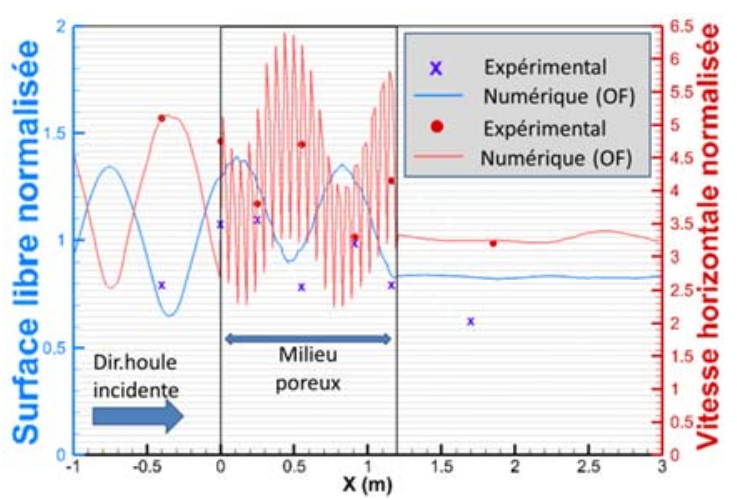

Figure 3. Résultats OpenFOAM de l'élévation de la surface libre et de la vitesse horizontale des particules comparés aux données expérimentales normalisées d'ARNAUD (2016).

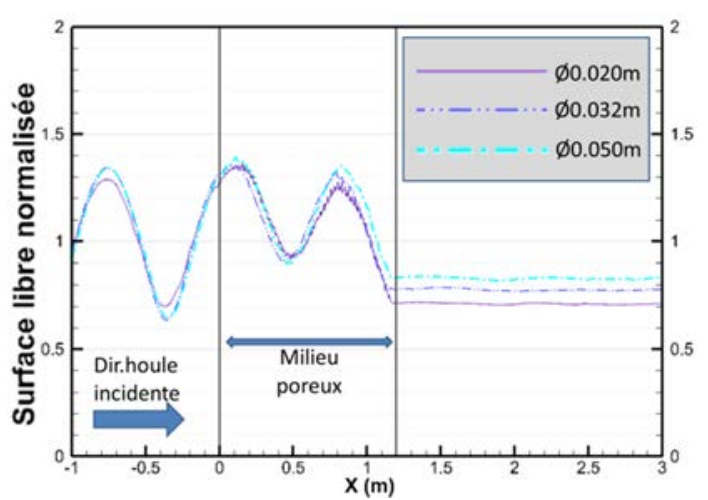

Figure 4. Surface libre normalisée obtenue en modélisation numérique OpenFOAM pour trois milieux poreux de différentes surfaces spécifiques soumis aux mêmes conditions de houle.

En amont et en aval du poreux l'amplitude des grandeurs est bien reproduite ce qui traduit la bonne appréciation par la modèle OF des coefficients de réflexion et transmission. Dans le poreux, la comparaison est plus délicate car les mesures sont trop difficiles et peu nombreuses pour traduire les fluctuations à l'échelle du motif. Le même niveau de résultat est obtenu pour toutes les combinaisons houle/poreux étudiées par 


\section{Thème 4 - Ouvrages portuaires, offshore et de plaisance}

ARNAUD. D'autres études portant sur le coefficient de traînée d'un cylindre au sein d'un réseau soumis à de la houle correspond à la littérature, notamment aux résultats de LIU et al. (2015). Cela confirme la validité du modèle, pas seulement par rapport aux données expérimentales d'ARNAUD (2016) mais également par rapport à d'autres travaux du domaine.

Si l'on considère que la houle ne déferle pas à l'entrée du milieu poreux, le coefficient de dissipation $\mathrm{C}_{\text {diss }}$ satisfait l'équation $\mathrm{C}_{\mathrm{r}}{ }^{2}+\mathrm{C}_{\mathrm{diss}}{ }^{2}+\mathrm{C}_{\mathrm{t}}{ }^{2}=1$, où : $\mathrm{C}_{\mathrm{r}}$ est le coefficient de réflexion, $\mathrm{C}_{\text {diss }}$ le coefficient de dissipation et $\mathrm{C}_{\mathrm{t}}$ le coefficient de transmission.

La figure 4 confirme le fait que la surface spécifique a une influence sur la transmission de la houle par le milieu poreux, bien que cette influence soit faible. En effet, chaque milieu poreux de 1.2 mètre de long de surface spécifique respective $s=21.82,33.51$, $52.36 \mathrm{~m}^{-1}$ induit un coefficient de dissipation de $0.43,0.52$ et 0.64 . Ces résultats correspondent aux résultats expérimentaux discutés dans le travail de recherche d'ARNAUD.

\subsection{Influence de la disposition et des paramètres de forme}

Dans cette partie, le modèle numérique précédemment validé est utilisé pour tester différentes dispositions des cylindres qui composent le milieu poreux ainsi que différentes géométries. Le but est d'étudier leur influence sur le taux de dissipation du milieu. Trois configurations différentes utilisant des cylindres de même diamètre $\mathrm{D}=0.032 \mathrm{~m}$ - sont comparées à la disposition initiale (i.e. coin en haut à gauche sur la figure 2). L'arrangement en ligne, perpendiculaire à la direction de propagation de la houle, affecte considérablement le coefficient de dissipation, tandis que les deux autres dispositions - coins en haut à droite et en bas à gauche sur la figure 2 - n'ont aucune influence notable sur les coefficients étudiés. Néanmoins, le temps de calcul est considérablement réduit lorsque les cylindres sont positionnés horizontalement, au lieu de verticalement. Les résultats étant quasi égaux pour ces deux configurations, il est intéressant de travailler avec la position horizontale pour réduire le temps de calcul. C'est le cas pour les deux dernières configurations de la figure 2, pour lesquelles la disposition ne change pas mais les cylindres sont transformés en éléments de section carrée, de face puis de biais par rapport à la direction de propagation de la houle.

La figure 5 donne une vue d'ensemble des coefficients de dissipation obtenus pour chacun de ces milieux poreux. Les structures étudiées ici font toutes 1.2 mètre de long et ont toutes les mêmes surface spécifique et porosité. La même houle - période de $1.2 \mathrm{~s}$, hauteur de vague de 0.017 mètre et profondeur d'eau de 0.23 mètre - leur est imposée. Cette figure confirme le fait que placer les cylindres horizontalement au lieu de verticalement - arrangements 1 (plan XY) et 4 (plan XZ) de gauche à droite sur la figure - n'affecte pas l'efficacité du milieu poreux, du moment que les calculs adéquats sont menés pour maintenir une surface spécifique et une porosité équivalentes. Afin 


\section{XVèmes Journées Nationales Génie Côtier - Génie Civil \\ La Rochelle, 29 au 31 mai 2018}

d'atteindre cette équivalence, il est souvent nécessaire de modifier le nombre d'éléments dans le réseau, tout en gardant une longueur globale de poreux de 1.2 mètre.
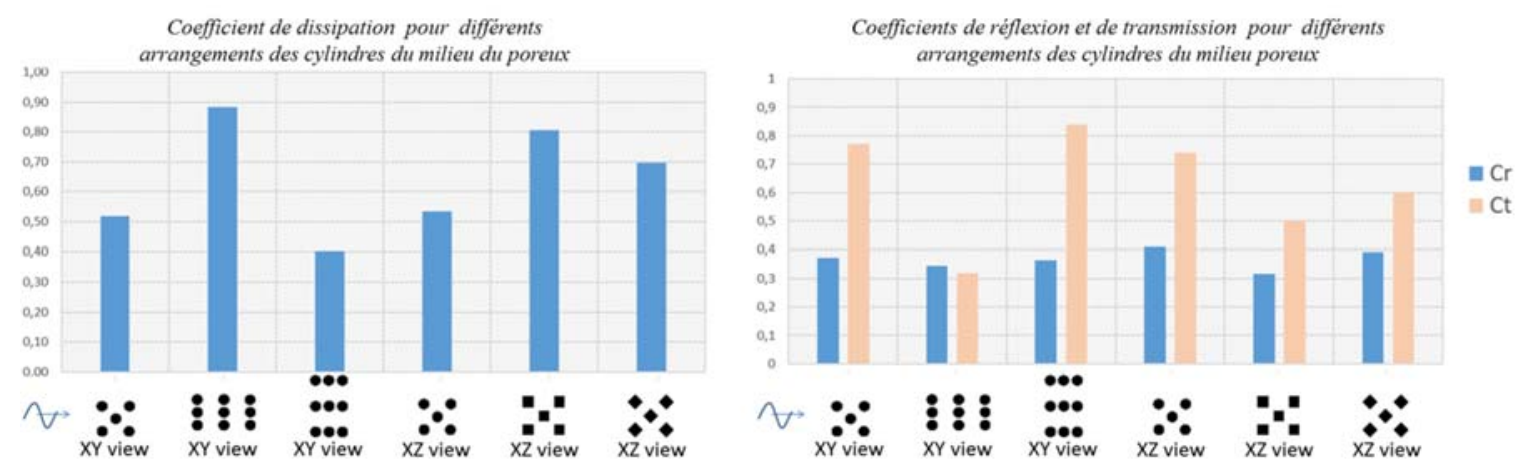

Figure 5. Coefficient de dissipation (à gauche) et coefficients de réflexion et de transmission (à droite) des différents milieux poreux testés dans le canal numérique. Sous les graphes, symboles des motifs des réseaux de cylindres tels que définis figure 2.

La figure 5 illustre que le simple fait de placer les cylindres différemment dans le réseau peut augmenter de $70 \%$ le taux de dissipation du milieu poreux, tout en gardant une même quantité de matériau et un même volume de structure. Cette observation est particulièrement intéressante du fait que le coefficient de réflexion reste plus ou moins constant (figure 5). Au regard de la dissipation, de la réflexion et de la transmission, la disposition numéro 2 semble bien meilleure que les autres. Cependant, il est nécessaire de réaliser une analyse des efforts hydrodynamiques sur la structure poreuse afin de savoir si les éléments du réseau résistent aux sollicitations. Dans cette configuration, les efforts seront sûrement plus importants puisque l'onde n'a que très peu d'espace pour franchir les rideaux de cylindres. Dans le modèle numérique, la force exacte exercée par l'eau sur un élément du réseau est difficile à évaluer précisément.

\section{Conclusion et perspectives}

Ce travail vise à approfondir les connaissances à propos de l'influence de certains paramètres caractéristiques des milieux poreux sur la capacité de ces derniers à dissiper de l'énergie. On étudie également la propagation de l'onde à l'intérieur de tels milieux. On montre que les résultats expérimentaux au sujet de l'influence de la surface spécifique, obtenus lors du travail de recherche d'ARNAUD (2016), sont à nouveau observés par notre modélisation de type RANS sans avoir recours à un calage spécifique. On montre ainsi la validité de ce type de modélisations pour approfondir la connaissance de la propagation de la houle au travers d'un milieu poreux. Ainsi, on profite de certaines facilités offertes par la modélisation numérique pour compléter les résultats déjà obtenus en canal à houle par ARNAUD (2016) en étendant l'étude à 


\section{Thème 4 - Ouvrages portuaires, offshore et de plaisance}

d'autres paramètres. La présente étude souligne le fait que l'importance de la surface spécifique ne peut être détachée de l'arrangement géométrique du poreux. On montre que pour une même surface spécifique, la disposition des motifs est déterminante pour la dissipation, principalement pour la transmission et relativement moins pour la réflexion. Ce point peut être important dans la conception d'ouvrage poreux. Il est toutefois nécessaire de préciser qu'en conception côtière, la plus grande dimension d'un ouvrage ne doit pas dépasser $10 \%$ de la longueur d'onde de la houle à laquelle elle est soumise. Ceci empêche la construction d'ouvrages protecteurs trop massifs dans la nature environnante. Ici, la longueur du milieu poreux représente près de $100 \%$ de la longueur d'onde de la houle à laquelle il est soumis. Une possible poursuite d'étude serait d'adapter la taille de la structure à des longueurs d'onde correspondant à des conditions réelles de houle. Le modèle développé ici serait un outil adéquat pour une telle étude. En effet, il permet de réaliser un modèle à l'échelle 1 et de se défaire ainsi de tout problème de similarité rencontré dans les modèles à échelle réduite présentant des effets visqueux. De plus, le canal numérique pourra être une solution efficace pour balayer certains paramètres adimensionnels permettant de caractériser l'interaction entre la disposition des éléments et la dissipation du milieu. Pour certaines configurations, nous avons également observé une modification de l'écoulement à l'intérieur du milieu poreux sous la forme de stratifications inattendues. La poursuite de l'étude, pourrait également permettre d'initier une analyse de ce phénomène pour définir la stratégie expérimentale à mettre en œuvre pour en valider l'existence. Enfin, cette étude a été focalisée sur la validation de la modélisation numérique des phénomènes physiques et le travail d'optimisation des temps de calcul a été négligé. Un effort devra être porté sur ce point afin de permettre des calculs avec des nombres d'éléments plus importants de façon augmenter la précision, réduire le nombre de symétries et ainsi prendre en compte les possibles effets de bord et interférences des sillages.

\section{Références}

ARNAUD G. (2016). Houle à la côte : propagation, impacts et ouvrages innovants. $\mathrm{PhD}$ thesis, Université de Toulon. URL : www.theses.fr/2016TOUL0009.pdf

CALHOUN R. J. (1971). Field study of wave transmission through a rubble-mound breakwater. United States Naval Postgraduate School. https://doi.org/10.5962/bhl.title.59791

LAMBERT R. J. (2012). Development of a numerical wave tank using OpenFOAM. $\mathrm{PhD}$ thesis University of Coimbra, Brazil. URL : https://estudogeral.sib.uc.pt/

LIU P. L. F., CHANG C. W., MEI C. C., LOMONACO P., MARTIN F. L., MAZA M. (2015). Periodic water waves through an aquatic forest. Coastal engineering 96(2015) 100-117. https://doi.org/10.1016/j.coastaleng.2014.11.002

MADSEN P. A. (1983). Wave reflection from a vertical permeable wave absorber. Coastal Engineering, Vol. 7, pp 381-396. https://doi.org/10.1016/0378-3839(83)90005-4 